\title{
DISEÑO E IMPLEMENTACIÓN DE UN CONTROL DUAL POR MODOS DESLIZANTES PARA UN CONVERTIDOR BUCK CD-CA
}

\section{DESIGN AND IMPLEMENTATION OF A DUAL SLIDING MODE CONTROL FOR A BUCK DC-AC CONVERTER}

Jaime Ayala Taco¹, Roberto Gutiérrez², Susset Guerra Jiménez ${ }^{3}$, Alexander Fernandez Correa ${ }^{4}$

Fecha de recepción: 23 de Septiembre de 2014

Fecha de aprobación: 26 de Noviembre de 2014

Referencia: J. Ayala Taco, R. Gutiérrez, S. Guerra Jiménez, A. Fernandez Correa. (2015). Diseño e implementación de un control dual por modos deslizantes para un convertidor Buck CD-CA. Ciencia e Ingeniería Neogranadina, 25 (1), pp. $91-111$

\section{RESUMEN}

El presente trabajo muestra un análisis orientado a la propuesta de diseño e implementación de un doble controlador en modo deslizante para un convertidor Buck CD-CA. Se plantean las superficies de deslizamiento candidatas de acuerdo con las exigencias de la aplicación y, mediante una lógica de activación de las llaves semiconductoras (MOSFET), se obtiene un control independiente en la amplitud de la tensión para el semiciclo positivo y negativo de la onda de salida en CA, con el objetivo de lograr a voluntad un nivel de CD introducido en la señal de alterna. Se ingresan perturbaciones de tensión en la entrada del convertidor y se producen variaciones en la carga con el objetivo de probar la robustez del sistema de control propuesto. El control se implementa en un microcontrolador AVR Atmega164PA y se prueba de manera práctica. Finalmente, se expone una comparación de los resultados obtenidos de manera experimental con las simulaciones realizadas en SCILAB5.

Palabras clave: Convertidor Buck CD-CA, modos deslizantes, control dual.

1. Ingeniero Electrónico, Profesor Depto. Eléctrica y Electrónica, Investigador. Universidad de las Fuerzas Armadas-ESPE, Quito, Ecuador, jpayala@espe.edu.ec

2. Ingeniero Mecánico, Profesor Depto. Energía y Mecánica, Investigador. Universidad de las Fuerzas Armadas-ESPE, Quito, Ecuador, ergutierrez@espe.edu.ec

3. Ingeniero Automática, Ph.D., Profesora-investigadora Depto. Automática. Instituto Politécnico José Antonio Echeverría, Habana, Cuba, suset@electrica.cujae.edu.cu

4. Ingeniero Electrónico, Ph.D., Profesor-investigador CIPEL. Instituto Politécnico José Antonio Echeverría, Habana, Cuba, alexandre@electrica.cujae.edu.cu 


\section{ABSTRACT}

This paper presents the design and implementation proposal of a dual sliding mode control for a Buck DC-AC converter. The candidate sliding surfaces were set out according to the demands of the application and, through a logic activation of the semiconductor keys (MOSFETs), independent control of the voltage amplitude for the positive and negative half cycles of the output wave in AC was obtained, in order to reach a specific level of DC introduced into the AC signal. Voltage disturbances were entered into the converter input and load variations were generated in order to test the robustness of the proposed control system. The control was implemented on a microcontroller AVR Atmega164PA and practically tested. Finally, a comparison of the results obtained experimentally and those obtained through simulations in SCILAB5 was made.

Keywords: DC-AC Buck Converter, sliding mode control, double control.

\section{INTRODUCCIÓN}

Los sistemas físicos tienen varias representaciones que permiten definir de una manera aceptable su comportamiento dinámico [1]. La necesidad de realizar un control por modos deslizantes surge debido a la característica primordial de los convertidores estáticos de ser sistemas de estructura variable [2], lo que se traduce como la variación de su comportamiento en función del estado de la llave semiconductora (conducción o no conducción).

El convertidor Buck permite transformar una tensión de entrada fija en una de salida regulada, con la particularidad de que aquella nunca será mayor a la tensión de entrada. Adicionalmente, esta magnitud siempre estará relacionada con el ciclo de trabajo D cuando el modo de operación es continuo. Para un convertidor reductor ideal se plantea que, cuando el ciclo de trabajo es del 50\%, la magnitud de la salida será la mitad de la de entrada. La particularidad del convertidor propuesto de ser inversor se produce debido a la presencia del puente $\mathrm{H}$ completo, que permitecambiarlatensión continuaenalterna [3]. El modo deslizante cumple la función de controlar el sistema mediante una lógica de conmutación de la llave semiconductora que se manipula en función de las ecuaciones diferenciales que caracterizan al sistema. Este puede ser presentado como la aplicación de una señal de control conmutando a una alta frecuencia, idealmente infinita, que conducirá la respuesta del sistema (trayectoria del comportamiento del régimen de estructura variable) hacia una superficie $\mathrm{S}=0$ denominada superficie de deslizamiento y, una vez alcanzada esta, debe mantenerse la trayectoria el resto del tiempo. De esta manera se pueden definir según [4-5] dos comportamientos en bucle cerrado:

- Modo de alcanzabilidad: Durante el cual la trayectoria de estado se mueve hacia la superficie de conmutación y la alcanza.

- Modo deslizante: Es el comportamiento en el que la trayectoria del estado se encuentra deslizando sobre la superficie. 
Los estados de conmutación de los MOSFET permiten realizar dos funciones específicas que son:

- La conversión de tensión continua a alterna, tomando en cuenta que la tensión alterna tiene una frecuencia de $60 \mathrm{~Hz}$ para la aplicación propuesta.

- El controlador por modo deslizante, que conmuta a una frecuencia máxima de 30 $\mathrm{kHz}$ y que permite tener una variación en tensión positiva y negativa.

En la literatura [6-7] se ha planteado el análisis de estabilidad y el empleo del controlador por modos deslizantes puros en aplicaciones de electrónica de potencia. Afsharian Zadeh et al. [8] presentan un controlador integral-derivativo deslizante para un convertidor Buck, con el que se obtienen resultados prometedores en cuanto a simulación. Finalmente, Yue et al. [9] proponen un control por modos deslizantes para un convertidor Buck, con el objetivo de alimentar una carga a potencia constante y verificar su robustez frente a variaciones en la carga.

La aplicación que se propone en el presente artículo establece la posibilidad de controlar de manera independiente las amplitudes positiva y negativa de la señal de CA de salida, con el objetivo de introducir un nivel de continua durante cierto número de ciclos en la carga. Esta señal puede ser empleada como perturbación del sistema con propósitos de identificación paramétrica sin alterar la frecuencia de operación de la planta.

\section{EL CONVERTIDOR BUCK CD-CA}

Este tipo de convertidor proporciona una tensión de salida regulada inferior a la de entrada. El estudio se realiza cuando el sistema se encuentra en estado continuo (modo de conducción continua, MCC), es decir, se realiza tomando en cuenta que la bobina siempre se mantiene cargada, nunca se descarga completamente, por lo que la corriente que pasa por la bobina siempre es mayor que 0 A [10]. La conmutación de los MOSFET del puente $\mathrm{H}$ es la que da origen al convertidor Buck. Como se observa en la Figura 1, el sentido de la corriente está dado por dos momentos de conmutación que son:

- Positivo: se produce cuando el MOSFET Q3 se encuentra desactivado y Q2 actúa como un diodo para el sentido positivo.

- Negativo: se produce cuando el MOSFET Q1 se encuentra desactivado $y$, en este caso, Q4 actúa como diodo, dando lugar a la tensión negativa en la carga.

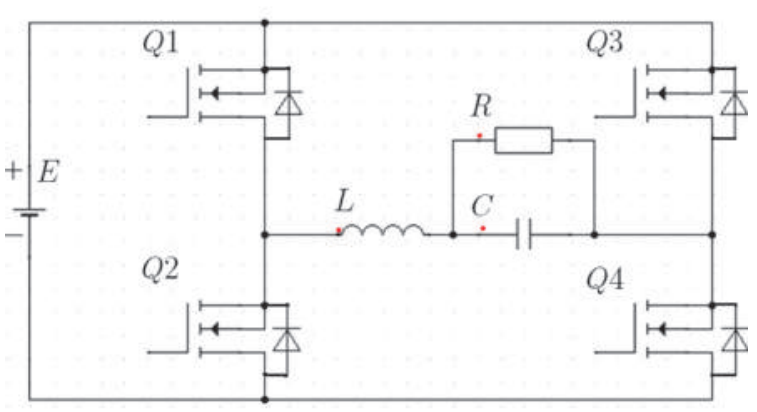

Figura 1. Puente completo en configuración $\mathrm{H}$.

Tomando en cuenta este tipo de conmutación, se obtiene la configuración típica de un convertidor Buck positivo y negativo. El sentido de la corriente depende propiamente del momento de conmutación de los MOSFET. Para el diseño y modelado del convertidor se propone que el sentido de la corriente sea positivo (en dirección de las agujas del reloj) y se toma como referencia que el MOSFET Q2 se 
comporta como un diodo, como se muestra en la Figura 2.

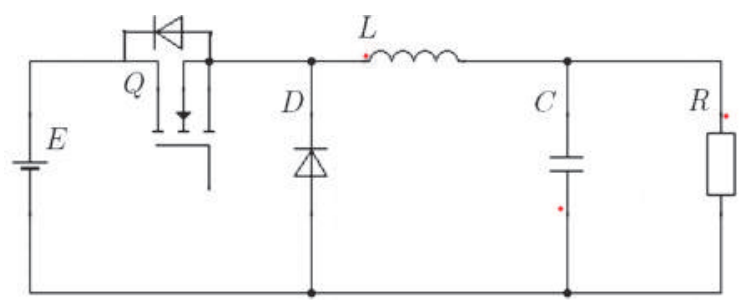

Figura 2. Convertidor Buck.

El sistema de estructura variable permite que la bobina trabaje siempre en estado continuo, como se observa en las Figuras 3 y 4 . En el primer periodo, el MOSFET Q1 se encuentra en conducción (estado ON), en el que la bobina se energiza por medio de la fuente $\mathrm{E}$ y el segundo periodo ocurre cuando el MOSFET Q1 se desactiva y la bobina transmite la energía a la carga por medio del diodo D [3].

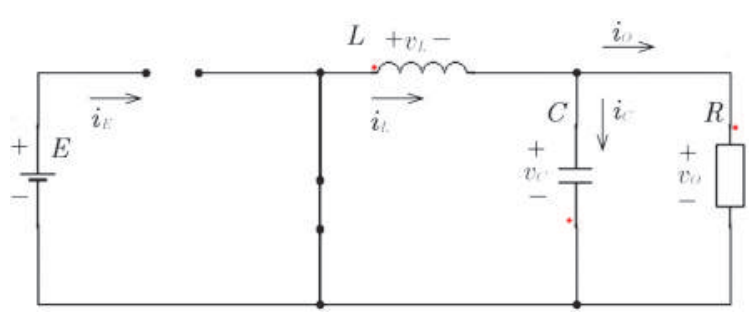

Figura 3. Estado $u=0$ en el convertidor.

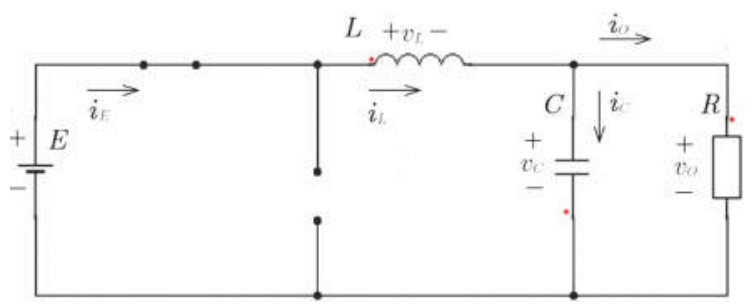

Figura 4. Estado $\mathrm{u}=1$ en el convertidor.
Las formas de onda del comportamiento deseado del convertidor se presentan en la Figura 5.

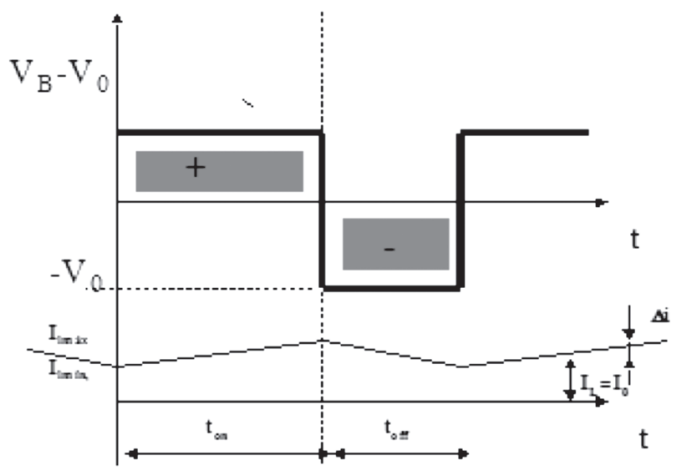

Figura 5. Formas de onda resultantes del convertidor.

Partiendo de las mismas, se obtienen las ecuaciones que describen la corriente y la tensión aplicables a este convertidor, cuando $T=T_{\text {ON }} \Rightarrow T=D . T[10]$.

$$
\begin{gathered}
V_{l}=E-V_{0} \\
I_{c}=I_{l}-I_{0} \\
I_{l}=I_{E} \\
R=\frac{V_{0}}{I_{0}}
\end{gathered}
$$

Conociendo el valor de la corriente en el inductor, se obtiene la ecuación (5).

$$
I_{l}=\frac{1}{L} \int V_{l} d t
$$


De las formas de onda de la Figura 5 surge el análisis para $T_{O N}=D . T$, donde $\mathrm{D}$ es el ciclo de trabajo, y se reemplaza el valor de la tensión del inductor de (1) en (5) para obtener la corriente.

$$
\Delta i_{l(+)}=\frac{1}{L} \int_{0}^{D T}\left(E-V_{0}\right) d t
$$

Se realiza la integral para $\Delta t[O-D . T]$ y se obtiene:

$$
\Delta i_{l(+)}=\frac{\left(E-V_{0}\right) \cdot D \cdot T}{L}
$$

En relación con la Figura 4 se obtienen las siguientes ecuaciones cuando $T=T_{\text {off }} \Rightarrow T=(1-D) T$

$$
\begin{gathered}
V_{l}=-V_{0} \\
I_{c}=I_{l}-I_{0}
\end{gathered}
$$

De las formas de onda de la Figura 5, se realiza el análisis para $T_{\text {off }}=(1-D) T$ y se reemplaza el valor de la tensión del inductor de (8) en (5).

$$
\Delta i_{l(-)}=\frac{1}{L} \int_{D T}^{0} V_{0} d t
$$

Al realizar la integral para (10) se obtiene:

$$
\Delta i_{l(-)}=\frac{V_{0} \cdot T \cdot(1-D)}{L}
$$

Cuando el convertidor se encuentra trabajando en régimen permanente se obtiene la siguiente relación:

$$
\begin{aligned}
\Delta i_{l(+)}=\Delta i_{l(-)} & \Rightarrow \frac{\left(E-V_{0}\right) \cdot D \cdot T}{L} \\
& =\frac{V_{0} \cdot T \cdot(1-D)}{L}
\end{aligned}
$$

Cuando se despeja $V_{0}$ de (12) se obtiene:

$$
V_{0}=D \cdot E
$$

La ecuación (13) es la que representa la tensión de salida $\left(V_{0}\right)$ en función de la de entrada $(E)$ que es directamente proporcional al ciclo de trabajo $(D)$, sin depender de ningún otro elemento del esquema. Para el análisis se han considerado despreciables las pérdidas asociadas a los elementos del circuito, por lo que:

$$
E . I_{E}=V_{0} \cdot I_{0}
$$

Se reemplaza el valor $V_{o}$ de (13) en (14) y al despejar $I_{E}$ se consigue:

$$
I_{E}=D \cdot I_{0}
$$

Esto significa que, en el modo de conducción continua, el convertidor reductor es equivalente a un transformador, en el que la relación de transformación será $D$. Para el diseño del inductor se despeja L en (7) y se obtiene: 


$$
L=\frac{\left(E-V_{0}\right) \cdot D \cdot T}{\Delta i_{l}}
$$

Se conoce que el teorema de valor medio es:

$$
I_{l} \geqq \frac{\Delta i_{l}}{2}
$$

Y además el valor de $T=1 / f_{s}$. Entonces, se reemplazan (17) y (13) en (16) y se consigue:

$$
L \geqq \frac{E \cdot D \cdot(1-D)}{2 I_{l} f_{s}}
$$

En (18) se reemplaza el valor de la I, de (15) y se consigue la relación en la bobina en función de la resistencia de carga, ciclo de trabajo y frecuencia de conmutación [10].

$$
L \geqq \frac{R(1-D)}{2 f_{s}}
$$

La ecuación (19) es la que servirá para el cálculo del valor del inductor. Si se supone que la componente ondulatoria de la corriente por la carga es muy pequeña y despreciable, entonces $\Delta i_{l}=\Delta i_{c}$. Teniendo en cuenta que la corriente media por el capacitor es cero, en el intervalo de $\Delta t[0, t / 2]$ la corriente media por el capacitor es $(\Delta i) / 4$ [11]. Conociendo el valor de la tensión en el capacitor se logra la ecuación (20).

$$
V_{c}=\frac{1}{C} \int \frac{\Delta I_{l}}{4} d t+V_{c}(0)
$$

Además se tiene que $\Delta V_{c}=V_{c}-V_{c}(0)$ y se consigue $\Delta V_{c}$ de (20). Al realizar la integral para $\Delta t[0, t / 2]$ se obtiene:

$$
\Delta V_{c}=\frac{\Delta I_{l}}{8 C f_{S}}
$$

Al reemplazar el valor de $\Delta I_{l}$ de (16) en (21) y al despejar el valor del capacitor (C) se consigue:

$$
C=\frac{E \cdot D \cdot(1-D)}{8 f_{s}^{2} L \Delta V_{c}}
$$

El rizado de tensión $\Delta V_{a^{\prime}}$ se puede expresar de la siguiente manera:

$$
\frac{\Delta V_{0}}{V_{0}} \leq X \%
$$

Cuando se toma en cuenta que $\Delta V_{0}=\Delta V_{c}$ y se reemplaza el rizado de tensión de (23) en (22), se consigue:

$$
C=\frac{E \cdot D \cdot(1-D)}{8 f_{s}^{2} L \cdot X \% \cdot V_{0}}
$$

\section{MODO DESLIZANTE APLICADO AL CONVERTIDOR BUCK CD-CA}

Para poder realizar el análisis del modo deslizante se debe partir desde el modelo matemático del convertidor Buck, tomando en cuenta las ecuaciones de estado para la tensión y la corriente. Para un sistema de estructura variable, como es el caso del convertidor, se 
tienen dos estados [5]: para cuando la señal de conmutación u toma un valor alto igual a uno y otro diferente cuando u toma un valor bajo igual a cero.

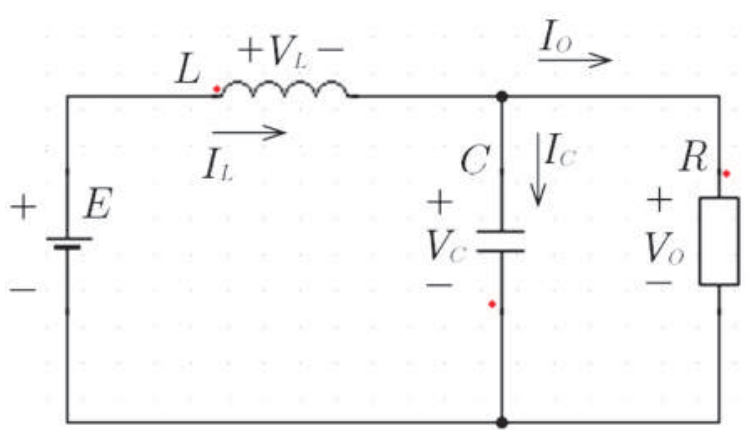

Figura 6. Diagrama de circuito en estado ON

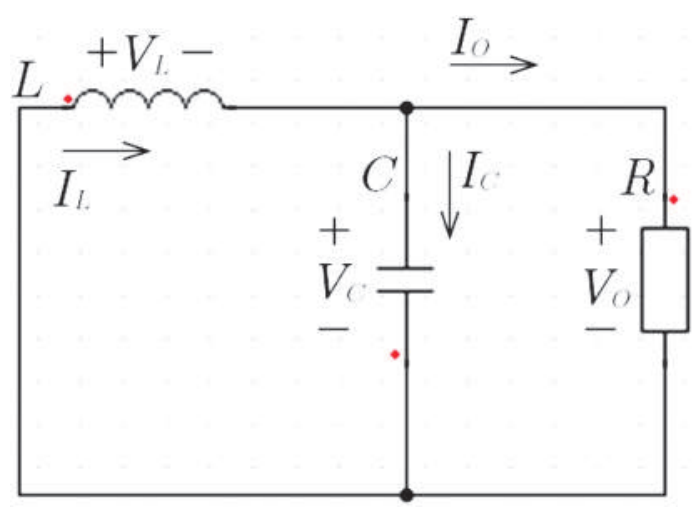

Figura 7. Diagrama de circuito en estado OFF.

Aplicando las leyes de Kirchhoff para el circuito de la Figura 7, se obtiene la ecuación para la corriente:

$$
I_{c}=-I_{0}+I_{l}
$$

Al saber que la corriente en el inductor es: $i_{c}=C \dot{V}_{c}$ y la corriente de salida es: $I_{0}=V_{c} / R$, se obtiene (26) reemplazando $I_{c}$ e $I_{o}$ en (25):

$$
C \dot{v}_{c}=\frac{-v_{c}}{R}+i_{l}
$$

Al despejar $v_{c}$ en (26), se obtiene la primera ecuación de estados en función de la derivada de la tensión del condensador:

$$
\dot{v}_{c}=\frac{-v_{c}}{R C}+\frac{1}{C} I_{l}
$$

Al aplicar las leyes de Kirchhoff para el circuito de la Figura 6, se tiene la ecuación para la tensión:

$$
V_{l}=E-V_{c}
$$

Al conocer que la tensión del inductor es: $V_{l}=L . i_{l^{\prime}}{ }^{\prime}$ se consigue (29) reemplazando V/en (28) :

$$
L i_{l}^{\prime}=E-v_{c}
$$

Cuando se despeja $i_{l}^{\prime}$ de (29), se obtiene la segunda ecuación de estados en función de la derivada de la corriente del inductor:

$$
i_{l}^{\prime}=-\frac{1}{L} v_{c}+\frac{E}{L} u
$$

Se realiza cambio de variable $\left(v_{c}=x_{1}\right.$ e $\left.i_{l}=x_{2}\right)$ y se expresan (27) y (30) en forma matricial.

$$
\begin{gathered}
\dot{x}=f(x)+g(x) u \\
{\left[\begin{array}{l}
\dot{x}_{1} \\
\dot{x}_{2}
\end{array}\right]=\left[\begin{array}{cc}
\frac{-1}{R C} & \frac{1}{C} \\
-\frac{1}{L} & 0
\end{array}\right]\left[\begin{array}{l}
x_{1} \\
x_{2}
\end{array}\right]+u\left[\begin{array}{l}
0 \\
\frac{E}{L}
\end{array}\right]}
\end{gathered}
$$


El sistema no lineal autónomo descrito por (31) cumple: $x \in X$, con $X \subset R^{n}$ conjunto abierto, es el vector de estados; $u: R^{n} \longrightarrow R$ que define la acción de control; $f$ y g son campos vectoriales locales suficientemente suaves y definidos en $X$, con $g(x) \neq 0, \forall x \in X[12-13]$.

Se define la función de conmutación h como una función suave $h: X \rightarrow R$, con gradiente dh no nulo en $X$, llamada también superficie de deslizamiento, que se debe proponer para ser sometida a validación. Para este caso en particular se plantea:

$$
S(x)=x_{2}-x_{2 r e f}=h(x)
$$

Donde $i_{l}=x_{2^{\prime}}$ es decir, la superficie de deslizamiento $S(x)$ dependerá del valor de corriente de la bobina y $x_{2 r e f}$ equivale a un valor constante ingresado como consigna del comportamiento deseado del sistema.

La ley de conmutación utilizada para un convertidor Buck dependerá del signo de la superficie de deslizamiento. Es decir, si $S(x)>0$, la ley de control forzará al vector de estados $u$ a ser 0 y, por el contario, si $S(x)<0$, la ley de control forzará al vector de estados u a ser 1 , donde $U^{+(x)}$ siempre será diferente de $U^{-(x)}[14]$, por lo que:

$$
\begin{aligned}
& u=\left\{\begin{array}{l}
1, \text { si } S(x) \geq 0 \\
0, \text { si } S(x)<0
\end{array}\right. \\
& u^{+}(x) \neq u^{-}(x)
\end{aligned}
$$

Se dice que existe un régimen deslizante sobre la superficie de deslizamiento cuando, como resultado de la ley de control, (33) el sistema alcanza dicha superficie (32) y se mantiene localmente en su entorno. Además, debe cumplirse que los campos vectoriales controlados $\left(f+g \cdot u^{+}\right)$y $\left(f+g \cdot u^{-}\right)$apunten localmente hacia la superficie de deslizamiento (ver Figura 8) [15].

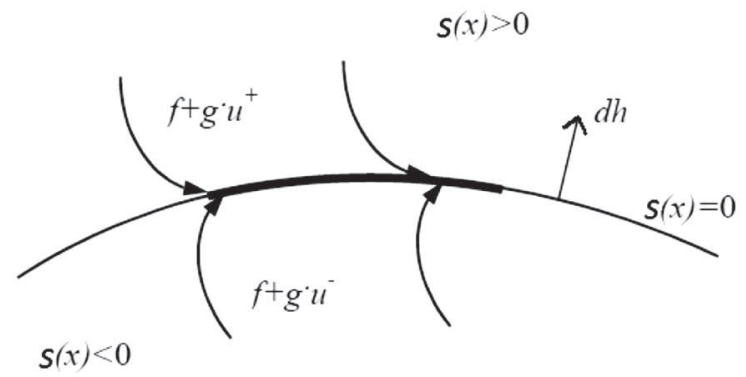

Figura 8. Régimen deslizante sobre una superficie de deslizamiento

Para esto se calcula la derivada direccional - llamada derivada de Lie, que denota la derivada parcial de un campo escalar $s: X \rightarrow R$ en la dirección de un campo vectorial $f(x): R^{n} \rightarrow R$ [15].

$$
\left\{\begin{array}{l}
L_{f} S(x)=\frac{\partial S}{\partial x_{1}} f_{1}+\frac{\partial S}{\partial x_{2}} f_{2} \\
L_{g} S(x)=\frac{\partial S}{\partial x_{1}} g_{1}+\frac{\partial S}{\partial x_{2}} g_{2}
\end{array}\right.
$$

Por lo tanto, considerando (31) se presentan los siguientes resultados parciales:

$$
\begin{aligned}
L_{f} S(x) & =0 \cdot\left(\frac{-x_{1}}{R C}+\frac{1}{C} x_{2}\right)+1 \cdot\left(-\frac{x_{1}}{L}\right) \\
& =-\frac{x_{1}}{L} 40 \\
L_{g} S(x) & =0 .(0)+1 \cdot\left(\frac{E}{L}\right)=\frac{E}{L}
\end{aligned}
$$


La región de convergencia se encuentra definida por (36), que se presenta en la Figura 9 [13].

$$
\begin{aligned}
& \mathbb{R}^{+}=\left\{x \in \mathbb{R}^{2}:<\nabla S, f(x)+u^{+} g(x)><0\right\} \\
& \mathbb{R}^{-}=\left\{x \in \mathbb{R}^{2}:<\nabla S, f(x)+u^{-} g(x)>>0\right\}
\end{aligned}
$$

$$
\left\{\begin{array}{c}
S(x)=0 \\
\dot{S}(x)=<\nabla S, f(x)+u_{e q} g(x)>=0
\end{array}\right.
$$

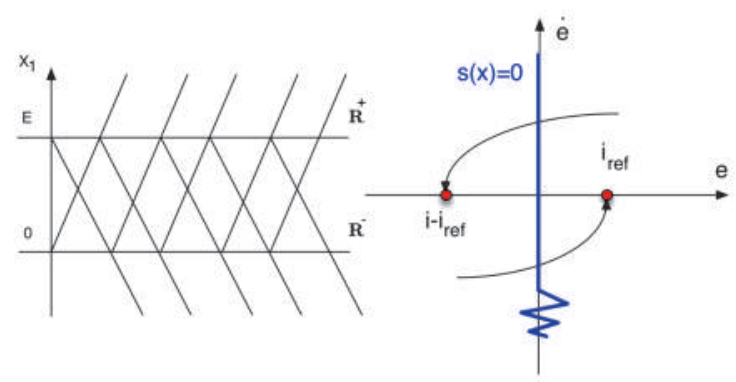

Figura 9. Región de convergencia.

Al conocer que el sistema conmuta a una alta velocidad idealmente infinita, se tiene un sistema discontinuo en todo el rango de tiempo. Para obtener una solución óptima se dispone del método del Control Equivalente $\left(u_{e d}\right)$ [14], que consiste en reconocer que $\dot{S}(x)=0$ es condición necesaria para confinar la trayectoria de estados a la superficie de deslizamiento $S(x)=0$. Esto se observa en la Figura 10.

Con base en lo expuesto, la condición de invariancia de la superficie de deslizamiento $S(x)$ estará dada por (37), y se obtendrá directamente el control equivalente mediante (38).

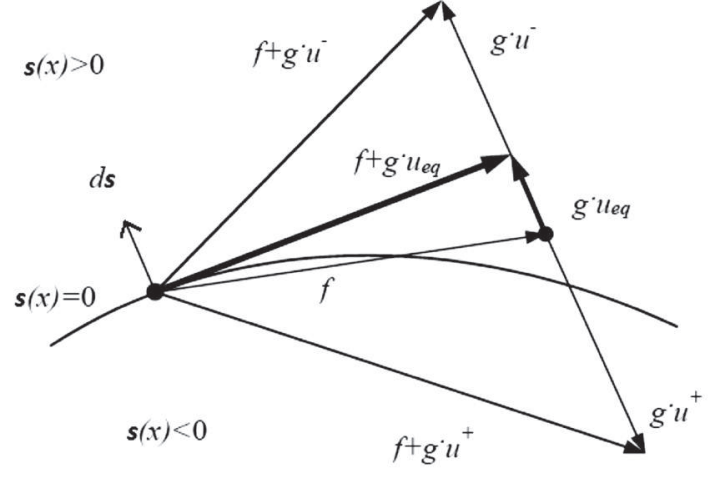

Figura 10. Método del control equivalente.

$$
u_{e q}=-\frac{L_{f} S}{L_{g} S}=\frac{x_{1}}{E}
$$

Por lo tanto, se puede decir que la condición suficiente para que exista un modo deslizante local en la superficie de deslizamiento es que se encuentre bien definida la $u$ [12] .

$$
\begin{aligned}
& 0<u_{e q}<1 \\
& 0<\frac{x_{1}}{E}<1
\end{aligned}
$$

El criterio de estabilidad usado para el análisis es el método directo de Lyapunov, debido a que permite analizar el sistema en su totalidad. Para ello, se considera la cantidad escalar definida por (40) como semipositiva (mayor o igual que cero), que representa cierta «energía» instantánea del error con respecto a la variedad $S$, que se iguala a cero sobre la misma [16].

$$
\varepsilon(x)=\frac{1}{2} S^{2}(x)
$$


Una estrategia recomendable para alcanzar, desde una vecindad de $S$, la condición deseable $h(x)=0$ es ejercer acciones de control $u \in[0,1]$, que resulten en un decrecimiento estricto de $\varepsilon(x)$ respecto al tiempo. Esto se logra influyendo sobre el sistema, de tal manera que la tasa de variación de $\varepsilon(x)$ respecto al tiempo sea estrictamente negativa [16]. Entonces, considerando (33) se obtiene la función de energía representada por (40).

$$
\dot{\varepsilon}(x)=S \dot{S}=S\left[L_{g} S\left(u-u_{e q}\right)\right]
$$

Al reemplazar (35) y (38) en (41) y considerando $u=1$ y $S=-S$ se obtiene la función de energía.

$$
\dot{\varepsilon}(x)=-S\left(\frac{E-1}{L}\right)<0
$$

Realizando lo propio y considerando $u=0$ y $S=S$ se obtiene:

$$
\dot{\varepsilon}(x)=-\left(\frac{S}{L}\right)<0
$$

Si se considera que los miembros de (42) y (43) todo el tiempo tienen valores positivos y que la tensión de entrada E siempre es mayor que uno, entonces se puede asegurar que la función de energía (40) hace asintóticamente estable al sistema (31) sobre la superficie de deslizamiento $S$ propuesta (32), confirmando su validez [13].

\section{LÓGICA DE FUNCIONAMIENTO PARA UN OFFSET}

El offset de tensión $\left(V_{\text {offset }}\right)$ en la carga se obtiene mediante un protocolo de conmutación para los MOSFET, de tal manera que se pueda tener un punto referencial o set point controlado para el ciclo positivo y un set point controlado para el ciclo negativo de manera independiente.

La configuración en puente $H_{1}$ Figura 1, permite tener 2 brazos que a su vez forman los 2 semiciclos de la señal $C A(f=60 \mathrm{~Hz})$, por lo que se ha divido a esta sección en 2 partes: semiciclo positivo comprendido entre [0-8.33] ms y semiciclo negativo comprendido entre [8.33-16.66] ms.

\subsection{SEMICICLO POSITIVO}

El semiciclo positivo ocurre cuando el transistor MOSFET Q3 está desactivado. El esquema de funcionamiento en estas condiciones se presenta en la Figura 11.

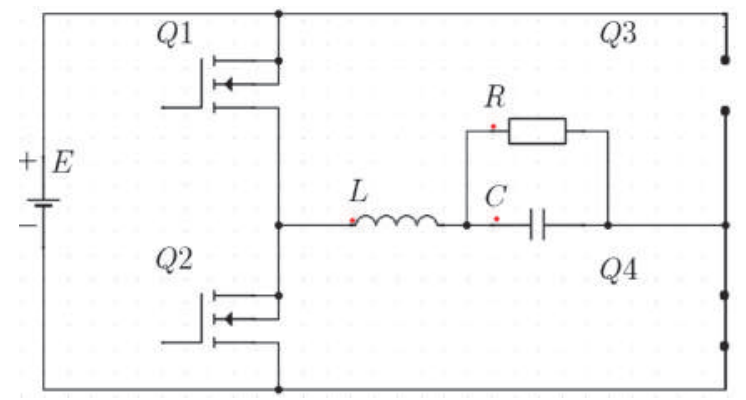

Figura 11. Puente $H$ semiciclo positivo.

Como se conoce, el convertidor Buck es un circuito de estructura variable [17]. Según sea la conmutación de los MOSFET se obtienen los dos estados del sistema, que son: a) cuando la bobina se energiza, Figura 6, y b) cuando la bobina entrega energía, Figura 7. Para obtener la configuración típica de un convertidor Buck, Figura 2, es necesario aplicar un protocolo de 
conmutación para el encendido y apagado de los MOSFET del semiciclo positivo.

- El primer estado se produce cuando la bobina se energiza, es decir, los MOSFET Q1 y Q4 estarán activados (ON) y por consecuencia los MOSFET Q2 y Q3 no lo estarán.

- El segundo estado se produce cuando la bobina entrega energía, es decir, los MOSFET Q2 y Q4 estarán activados (ON) y por consecuencia los MOSFET Q1 y Q3 no lo estarán.

La activación y desactivación de los transistores MOSFET forman el protocolo de conmutación para el semiciclo positivo, como se muestra en la Tabla 1.

Tabla 1. Lógica de conmutación semiciclo positivo.

\begin{tabular}{|c|c|c|c|}
\hline Q1 & Q2 & Q3 & Q4 \\
\hline ON & OFF & OFF & ON \\
\hline OFF & ON & OFF & ON \\
\hline
\end{tabular}

Para este semiciclo, los MOSFET Q1 y Q2 conmutan inversamente a una frecuencia no mayor a 30 kHz, como se observa en la Figura 13. La amplitud de la tensión del semiciclo positivo depende propiamente del tiempo de carga y descarga del inductor. Es decir, si el MOSFET Q1 está un mayor tiempo encendido con relación a Q2, la bobina se carga por un mayor intervalo y la tensión es superior. Por el contrario, si el MOSFET Q1 se encuentra un menor tiempo encendido con relación a Q2, la bobina se carga por un instante pequeño y la tensión es inferior.

El control de modo deslizante del semiciclo positivo se propone para la conmutación de los MOSFET Q1 y Q2.

\subsection{SEMICICLO NEGATIVO}

El semiciclo negativo ocurre cuando el transistor MOSFET Q1 está desactivado. El esquema de funcionamiento en estas condiciones se presenta en la Figura 12.

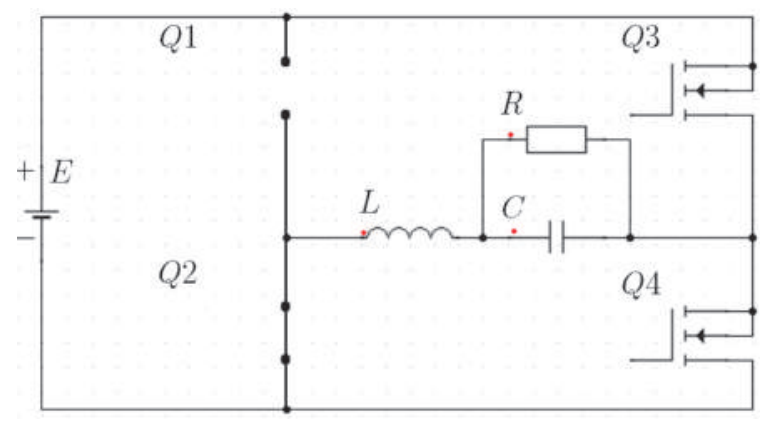

Figura 12. Puente H semiciclo negativo.

El ciclo negativo de la Figura 12 se analiza de la misma manera que el ciclo positivo, tomando en cuenta los dos estados del circuito de estructura variable:

- El primer estado se produce cuando la bobina se energiza, es decir, los MOSFET Q3 y Q2 estarán activados (ON) y por consecuencia los MOSFET Q1 y Q4 no lo están.

- El segundo estado se produce cuando la bobina transmite, es decir los MOSFET Q2 y Q4 estarán activados (ON) y por consecuencia los MOSFET Q1 y Q3 no lo estarán.

La activación y desactivación de los transistores MOSFET forman el protocolo de conmutación para el semiciclo negativo, como se muestra en la Tabla 2. 
Tabla 2. Lógica de conmutación semiciclo negativo.

\begin{tabular}{|c|c|c|c|}
\hline Q1 & Q2 & Q3 & Q4 \\
\hline OFF & ON & ON & OFF \\
\hline OFF & ON & OFF & ON \\
\hline
\end{tabular}

Para este caso, Q1 y Q2 no cambian de estado, Q3 y Q4 estarán conmutando a una frecuencia no mayor a $30 \mathrm{kHz}$. Es decir que según el ciclo de trabajo que tengan los MOSFET Q3 y Q4 depende la amplitud de la tensión en el semiciclo negativo, tomando en cuenta que conmutan inversamente como se muestra en la Figura 13.

El control por modo deslizante del semiciclo negativo se propone para la conmutación de los MOSFET Q3 y Q4.
La unión del funcionamiento de los semiciclos positivo y negativo permite obtener el protocolo de conmutación de $V_{\text {offset }}$

El protocolo de conmutación de los MOSFET depende propiamente del funcionamiento individual de cada uno de ellos, y su frecuencia varía entre $30 \mathrm{kHz}$ y $60 \mathrm{~Hz}$, además de su ciclo de trabajo.

Para obtener un offset de tensión ( $\left.V_{\text {offset }}\right)$ hay que tomar en cuenta que set point del semiciclo positivo debe ser diferente al del semiciclo negativo, es decir que el ciclo de trabajo debe ser diferente para los dos casos. Para obtener un offset positivo es necesario que el set point de tensión del semiciclo positivo sea mayor al del negativo y para obtener un offset negativo se necesita todo lo contrario.

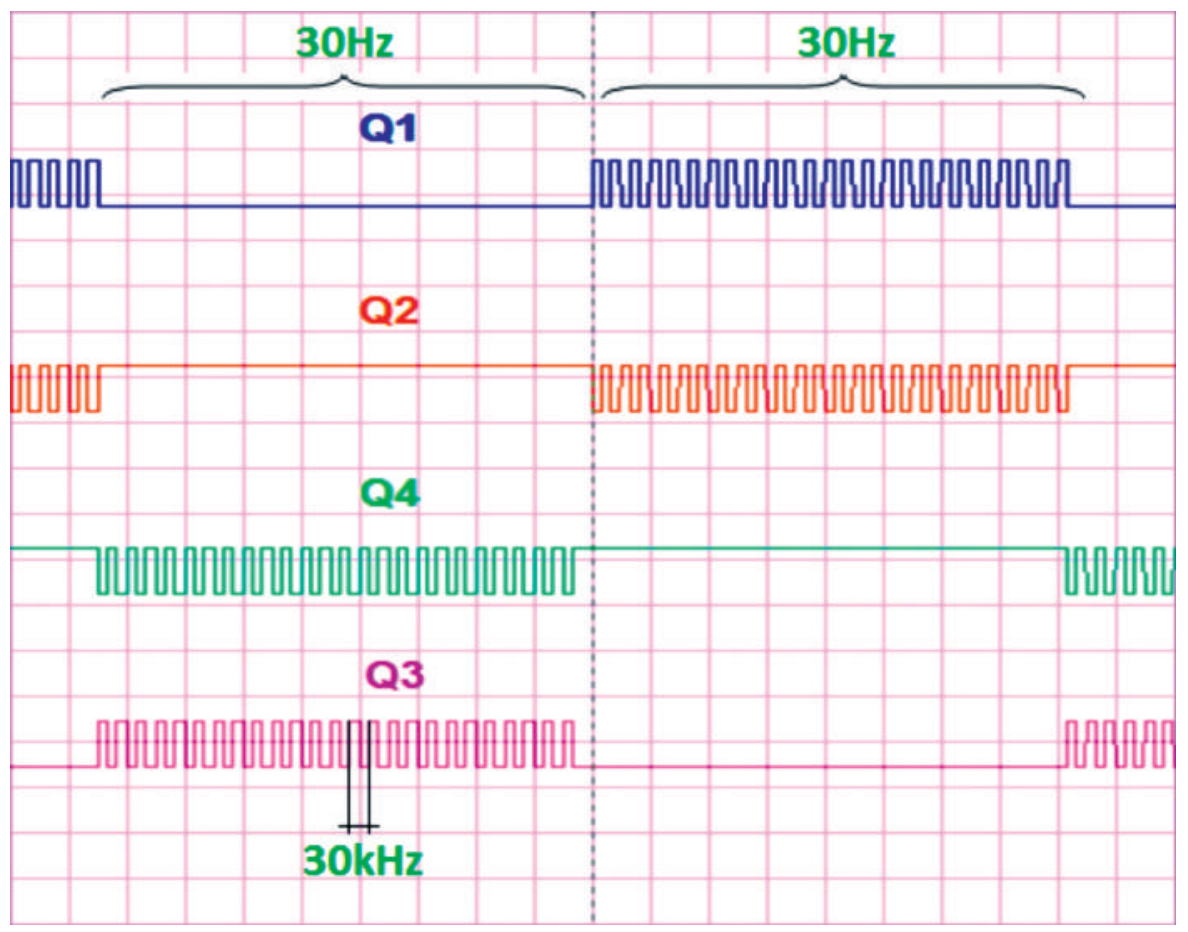

Figura 13. Lógica de conmutación de V_offset. 


\section{SIMULACIÓN}

La simulación se realiza utilizando el paquete SCILAB 5. Para obtener una respuesta en el tiempo de las variables de tensión y corriente se ejecutan dos scripts.

- El primero es el programa que define los parámetros propios del convertidor Buck, es decir, $\mathrm{L}=1 \mathrm{mH}, \mathrm{E}=50 \mathrm{~V}, \mathrm{R}=200 \Omega, \mathrm{y} \mathrm{C}=$ 2, 6 uF.

- El segundo es el programa del controlador en el que las variables de estado se acercarán a la superficie de deslizamiento $S$ y se mantienen al llegar al estado permanente. Es necesario recalcar que la frecuencia de conmutación depende del control por modos deslizantes [11].

La simulación se realiza para un medio periodo, tomando en cuenta que el semiciclo negativo es similar.

En la Figura 14a se observa la respuesta de la corriente, que llega al valor deseado que, en este caso, es de $2 \mathrm{~A}$ y luego se mantiene en este valor. El controlador de modo deslizante es robusto $y$, como se observa en la Figura $14 a$, el sistema alcanza la superficie de deslizamiento en una forma casi inmediata. En el proceso de simulación no se ha empleado una frecuencia infinita, ya que la misma es imposible dadas las limitaciones de los semiconductores, por lo que es notable en la Figura 14b la presencia de una histéresis de corriente, comúnmente conocida como chattering [14]. En la implementación se ha adoptado una estrategia de limitación por banda para reducir el efecto de este fenómeno.

Para el proceso de implementación, es necesario depender de la variable de tensión que se encuentra reflejada con la variable de corriente, tomando en cuenta que la planta siempre es la misma.

La Figura 15a muestra la respuesta transitoria de la tensión que, al igual que la corriente, alcanza su valor deseado y se desliza sobre

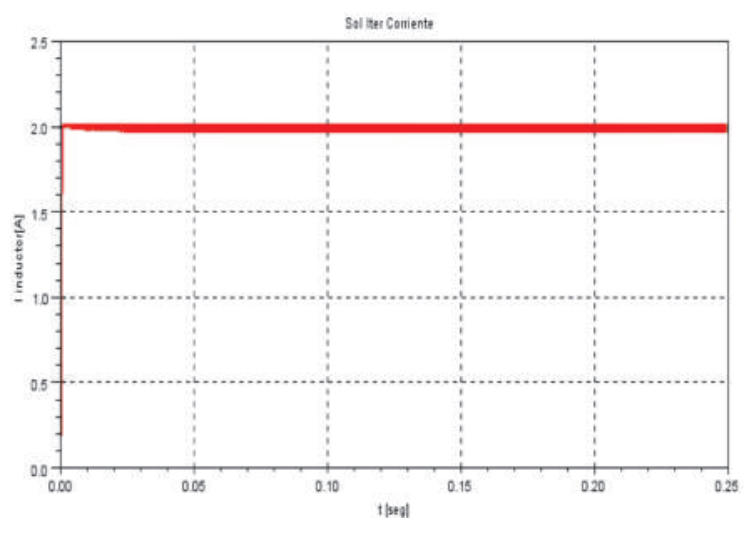

(a) Respuesta Transitoria Corriente.

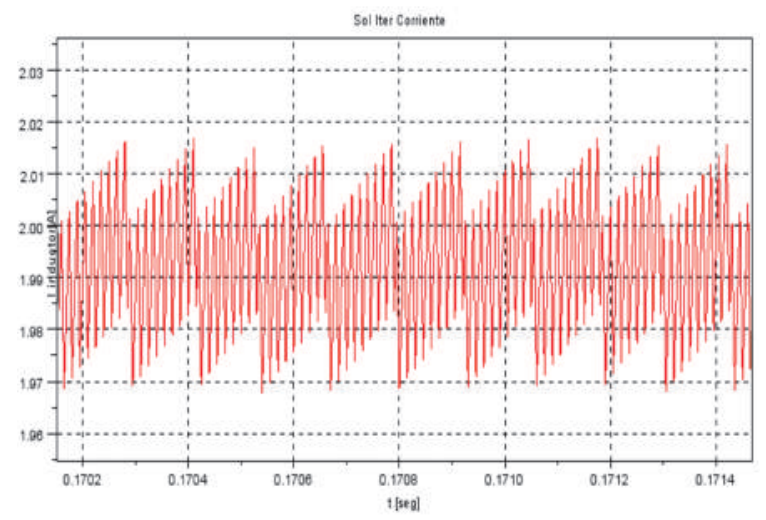

(b) Histéresis de la corriente.

Figura 14. Corriente en el inductor con el control de modo deslizante. 


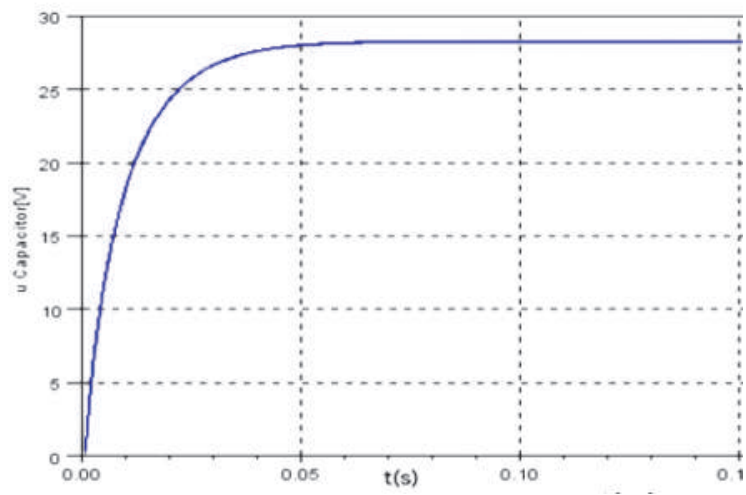

(a) Respuesta transitoria de la tensión controlada.

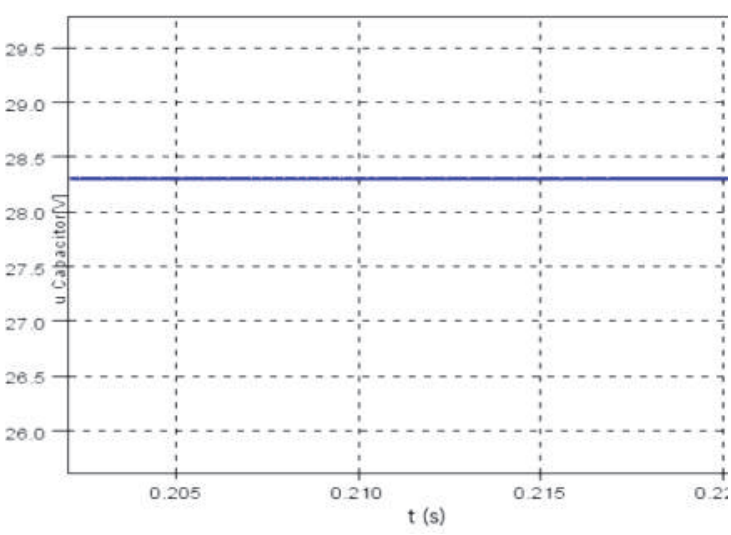

(b) Histéresis de tensión.

Figura 15. Corriente en el inductor con el control de modo deslizante.

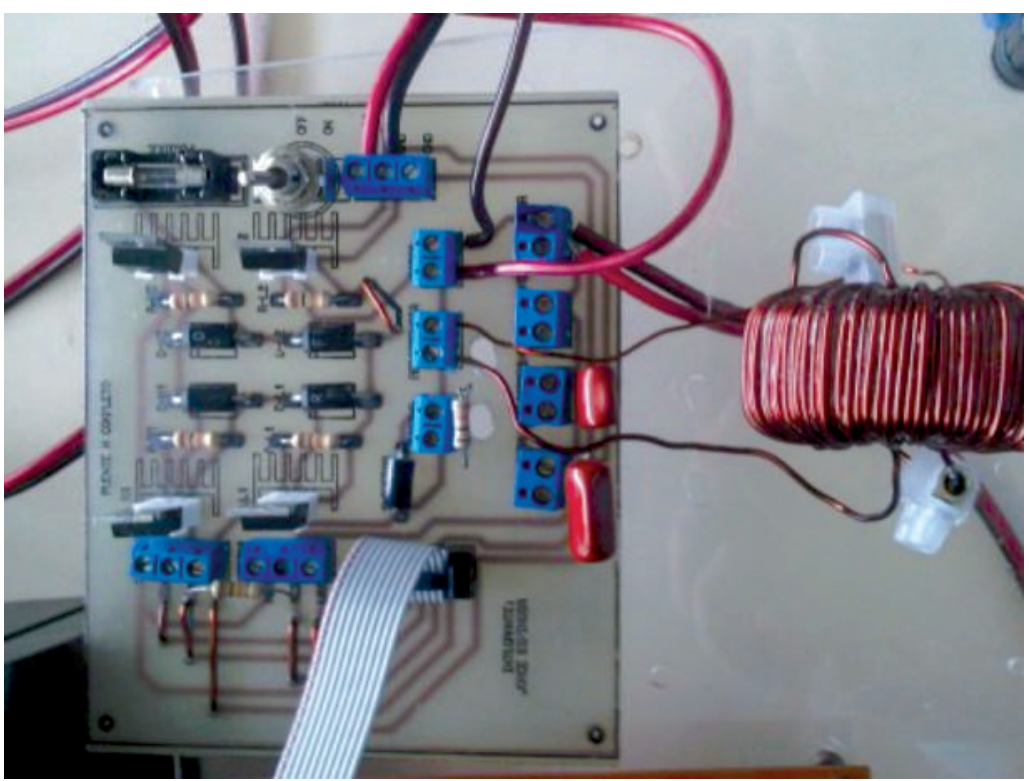

Figura 16. Circuito convertidor Buck implementado

dicho valor. En el caso de la tensión, esta alcanza un valor aproximado de $28 \mathrm{~V}$. Es posible afirmar, entonces, que el sistema se puede controlar por tensión de una forma indirecta en función de la corriente.

\section{IMPLEMENTACIÓN}

El diseño de los circuitos electrónicos implementados se realizó en tres etapas y cada una se construyó en una tarjeta electrónica diferente. 


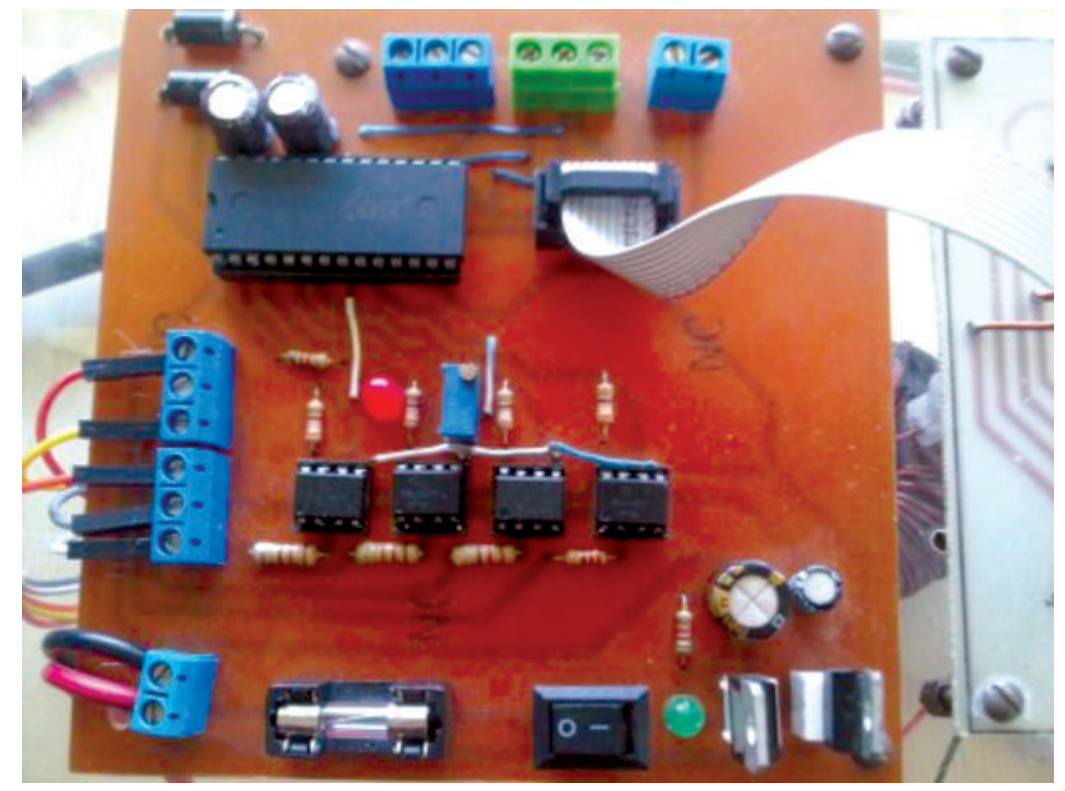

Figura 17. Circuito interfaz de potencia implementado

La primera corresponde a la construcción del La segunda etapa es la construcción del puente $\mathrm{H}$ y la carga, es decir, de la planta o circuito electrónico (IR2130) que controla circuito de potencia. los transistores MOSFET (IRF1310N), los

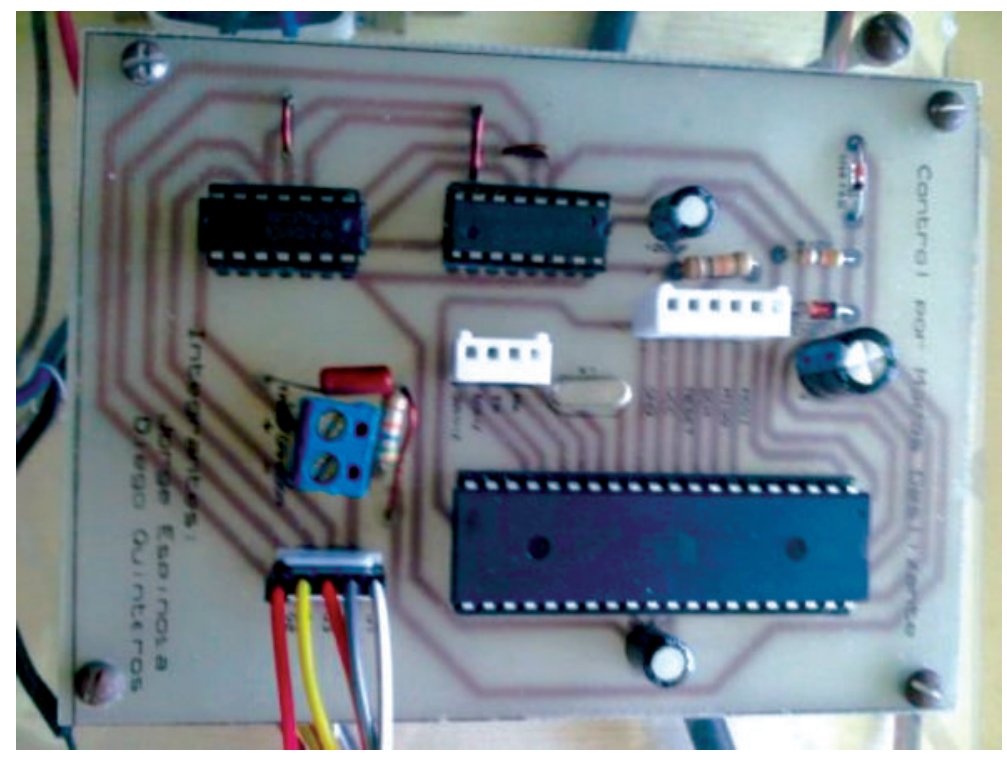

Figura 18. Circuito de control implementado. 
cuales forman parte integral del circuito de potencia.

La tercera etapa implica la construcción del circuito de control, que produce el protocolo de conmutación para los MOSFET. Aquí se encuentra el microcontrolador AVR Atmega164PA y, además, los filtros de tratamiento de la señal de sensado de tensión por medio de un transformador con relación 10:1 y el sensor de intensidad ACS712.

Adicionalmente se emplea un reóstato de 0 a $440 \Omega, 1.8$ A como carga del sistema.

\section{RESULTADOS}

\subsection{PRIMERA PRUEBA}

El punto referencial o set point es una corriente circulante en el inductor de $0.034 \mathrm{~A}$, para una tensión en la salida de $12.1 \mathrm{~V}$.

\subsubsection{Simulación}

Se observa en la Figura 19a que se alcanza el punto de estabilidad de la corriente en el inductor alrededor de $0.034 \mathrm{~A}$ y con un rango de histéresis entre [0.029-0.041] $A$, aproximadamente. En tensión, la estabilización se produce en $12.1 \mathrm{~V}$.

\subsubsection{Funcionamiento práctico}

Una vez colocado en el programa desarrollado en el microcontrolador el valor referencial de $0.034 \mathrm{~A}$ para una tensión en la salida de $12.1 \mathrm{~V}$, se enciende el sistema controlado y se obtiene la imagen de la Figura 19b, la cual muestra una estabilización de la corriente y tensión de $0.031 \mathrm{~A}$ y $11.3 \mathrm{~V}$, respectivamente.

\subsubsection{Cálculo de errores}

- Corriente:

$$
\begin{aligned}
\text { Error } & =\left|\frac{I_{\text {simulada }}-I_{\text {práctica }}}{I_{\text {simulada }}}\right| 100 \\
& =\left|\frac{0.034-0.031}{0.034}\right| 100=8.82 \%
\end{aligned}
$$

- Tensión:

$$
\begin{aligned}
\text { Error } & =\left|\frac{V_{\text {simulada }}-V_{\text {práctica }}}{V_{\text {simulada }}}\right| 100 \\
& =\left|\frac{12.1-11.3}{11.3}\right| 100=6.61 \%
\end{aligned}
$$

\subsection{SEGUNDA PRUEBA}

El punto referencial o set point es una corriente circulante en el inductor de $0.235 \mathrm{~A}$, para una tensión en la salida de 31.3 V.

\subsubsection{Simulación}

Se observa en la Figura 20a que se alcanza el control de la corriente en el inductor alrededor de $0.235 \mathrm{~A}$ y con un rango de histéresis entre [0.215-0.255] A, aproximadamente. En tensión, la estabilización se produce alrededor de $31.3 \mathrm{~V}$.

\subsubsection{Funcionamiento práctico}

Una vez colocado en el programa del microcontrolador el valor referencial de 0.235 A para una tensión en la salida de $31.3 \mathrm{~V}$, se enciende el sistema controlado y se obtiene la imagen de la Figura 20b, la cual muestra una estabilización de la corriente y tensión de 0.228 A y $30 \mathrm{~V}$, respectivamente. 

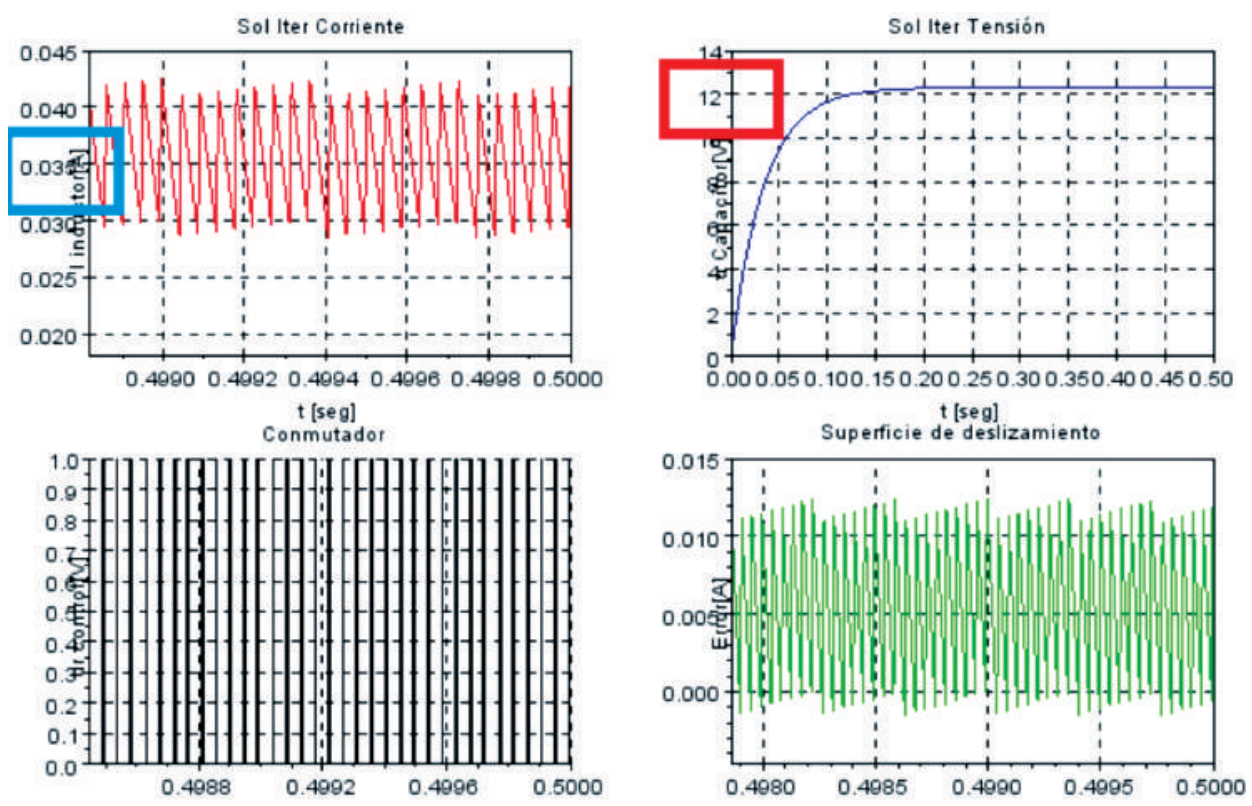

(a) Simulación

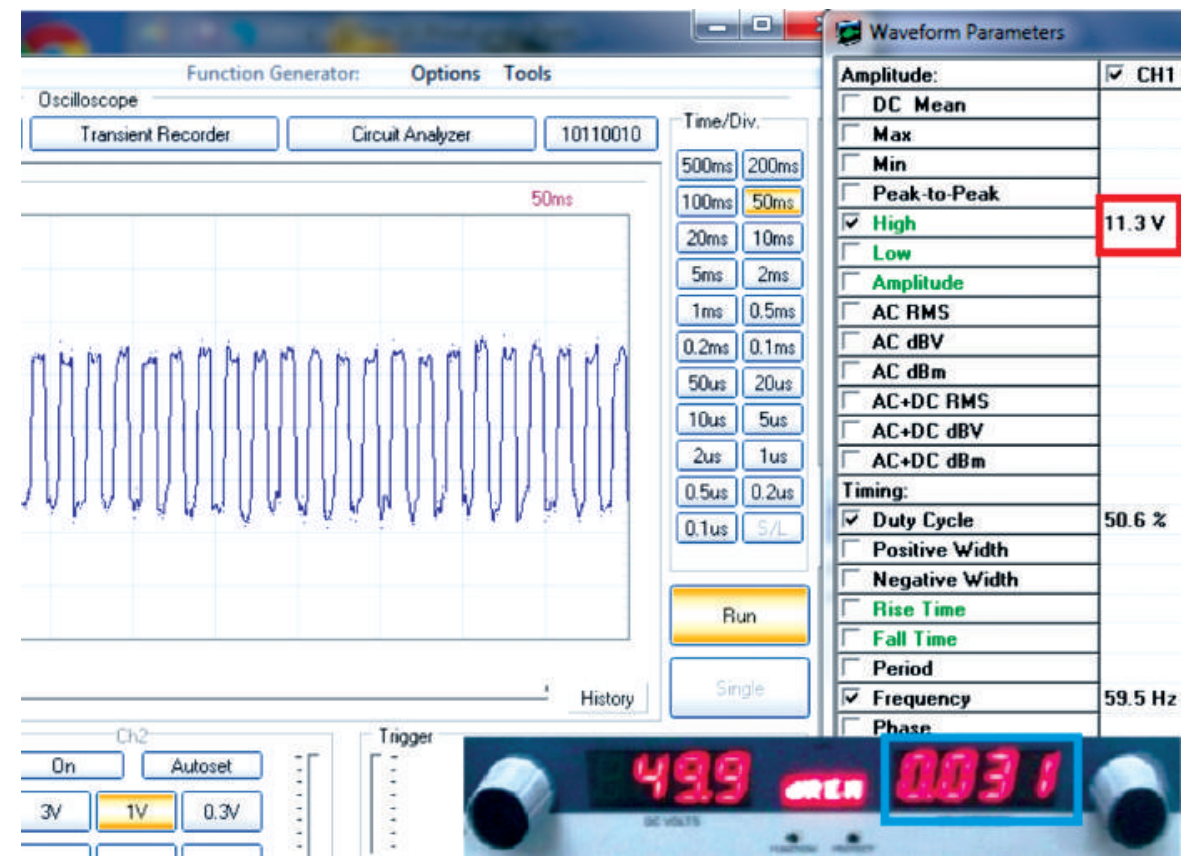

(b) Funcionamiento Práctico

Figura 19. Sistema controlado a $0.034 \mathrm{~A}$ y $12.1 \mathrm{~V}$. 

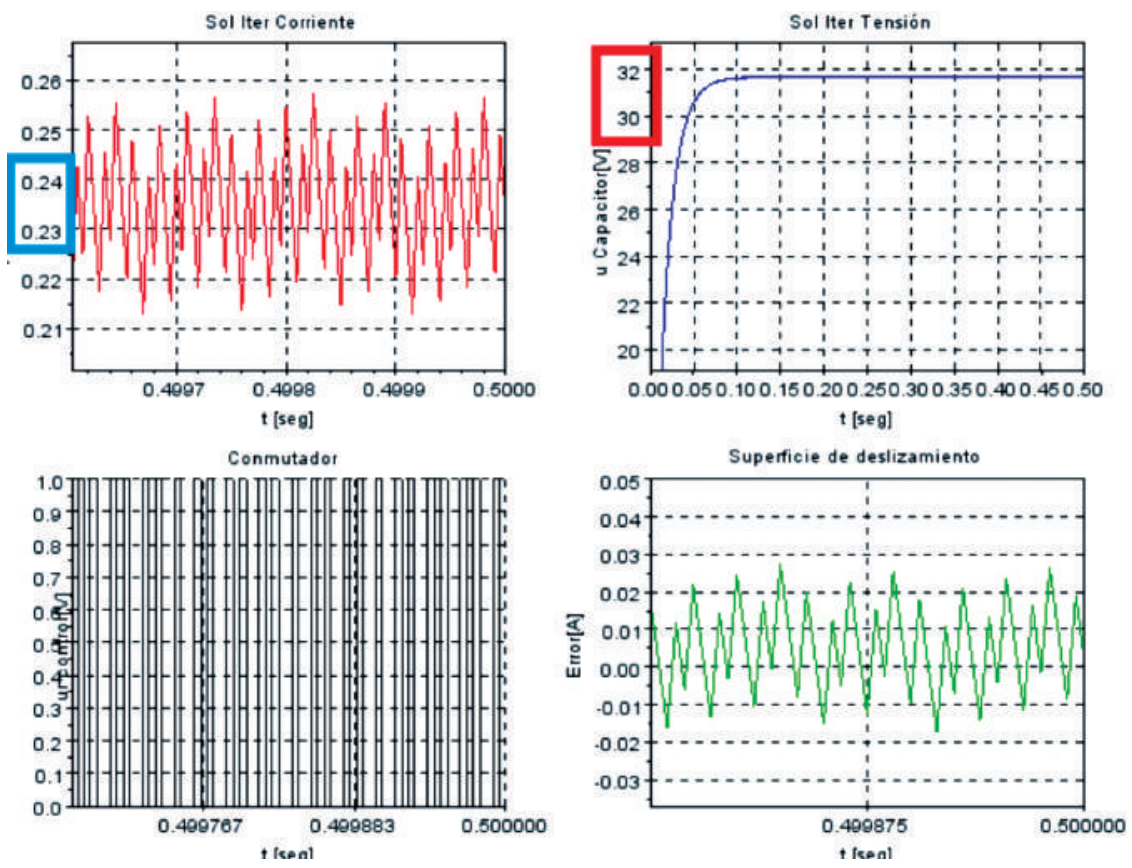

(a) Simulación

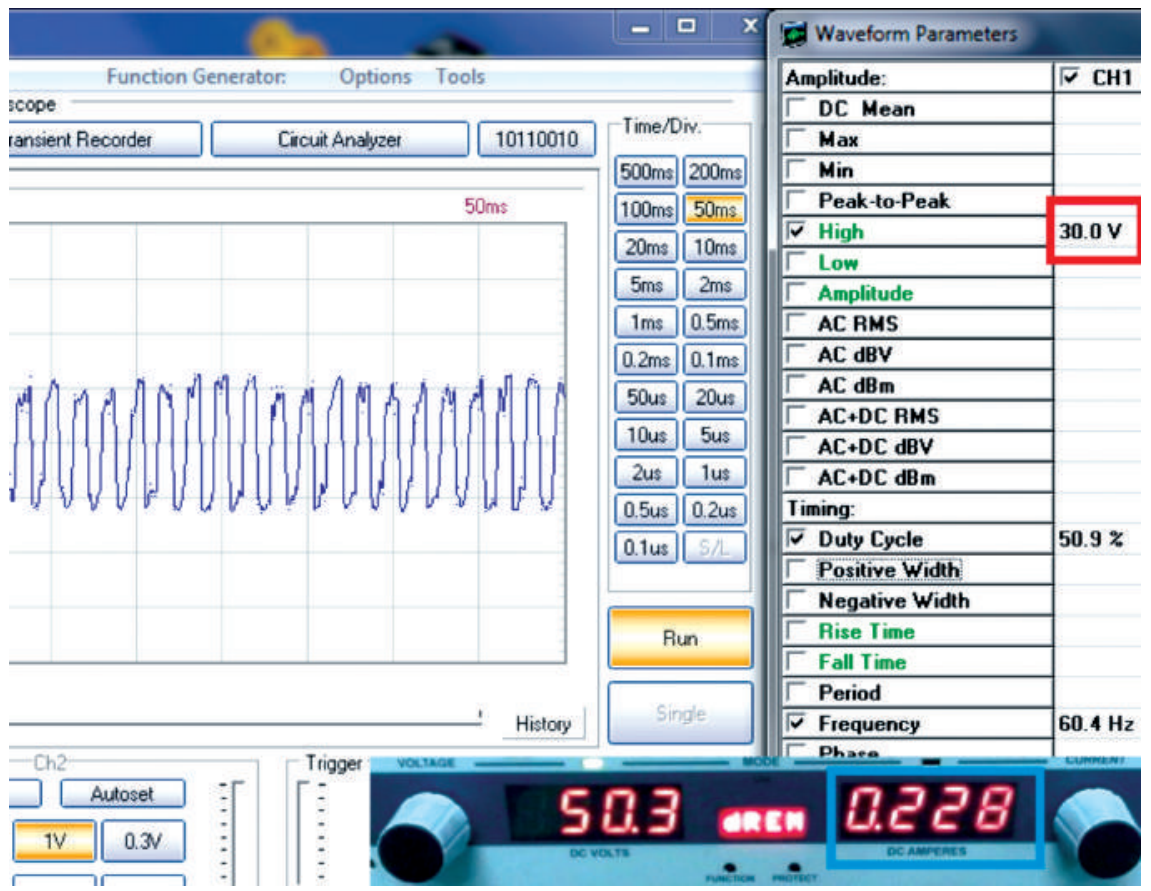

(b) Funcionamiento Práctico

Figura 20. Sistema controlado a 0.235 A y $31.3 \mathrm{~V}$. 


\subsubsection{Cálculo de errores}

- Corriente:

$$
\begin{aligned}
\text { Error } & =\left|\frac{I_{\text {simulada }}-I_{\text {práctica }}}{I_{\text {simulada }}}\right| 100 \\
& =\left|\frac{0.235-0.228}{0.235}\right| 100=2.97 \%
\end{aligned}
$$

- Tensión:

$$
\begin{aligned}
\text { Error } & =\left|\frac{V_{\text {simulada }}-V_{\text {práctica }}}{V_{\text {simulada }}}\right| 100 \\
& =\left|\frac{31.3-30.0}{31.3}\right| 100=4.15 \%
\end{aligned}
$$

\subsection{ANÁLISIS DE ERRORES}

Los errores se presentan debido a que en la simulación se trabaja con valores ideales de capacitancia, inductancia y resistencia. Todos estos elementos tienen una tolerancia, además de una pérdida de energía transformada en calor. Además de ello, no se han considerado las pérdidas de conmutación en las llaves semiconductoras.

El error promedio en la tensión del capacitor es $5.45 \%$. Esto se produce por 2 aspectos: primero, por la tensión de polarización de los diodos, que para el caso del semiconductor FR307 es $1.2 \mathrm{~V} y$, en segundo lugar, por la resistencia parásita que se encuentra en serie al inductor $\mathrm{L}$.

El error promedio en la corriente que circula por el inductor es $5.67 \%$. Esto se debe al rizado de carga y descarga de la bobina que produce una histéresis en la corriente. Este efecto, al ser filtrado y rectificado en el proceso de sensamiento, impide tener un dato exacto que represente su magnitud real.
Al aplicar una perturbación para el caso de funcionamiento con un set point de $0.235 \mathrm{~A}$ sobre la resistencia de carga $( \pm 20 \%$ de su valor nominal de operación de $200 \Omega$ ), la variación en la corriente de salida prácticamente es imperceptible, es decir, el control de modo deslizanteactúaóptimamenteaperturbaciones en la carga. Conforme se presenta en la Figura 21 para el caso de una carga de $180 \Omega$, se obtiene una tensión de $27.2 \mathrm{~V}$ en la salida, lo que corresponde a una corriente en estado estable de $0.228 \mathrm{~A}$, prácticamente igual al de la prueba de funcionamiento presentada en la Figura 20b.

\section{CONCLUSIONES}

La característica más significativa del control de modo deslizante es su robustez a estímulos externos, lo que se demostró experimentalmente al producir variaciones físicas en la resistencia de carga y la tensión de entrada.

Paralaelección de lasuperficie de deslizamiento se probaron algunas superficies candidatas, pero se debe tomar en consideración una superficie que cumpla con las condiciones de existencia, transversalidad y cumpla con los criterios de estabilidad por el método de Lyapunov.

El offset de tensión se puede obtener al invertir los ciclos de trabajo de los dos semiciclos de la onda alterna, es decir, cuando el ciclo de trabajo del semiciclo positivo sea del $70 \%$, el ciclo de trabajo del semiciclo negativo es del $30 \%$. Esto significa que la tensión positiva es mayor que la tensión negativa y, por esta razón, existe un offset de tensión. Este cambio del ciclo de trabajo se realiza en el protocolo de conmutación. 


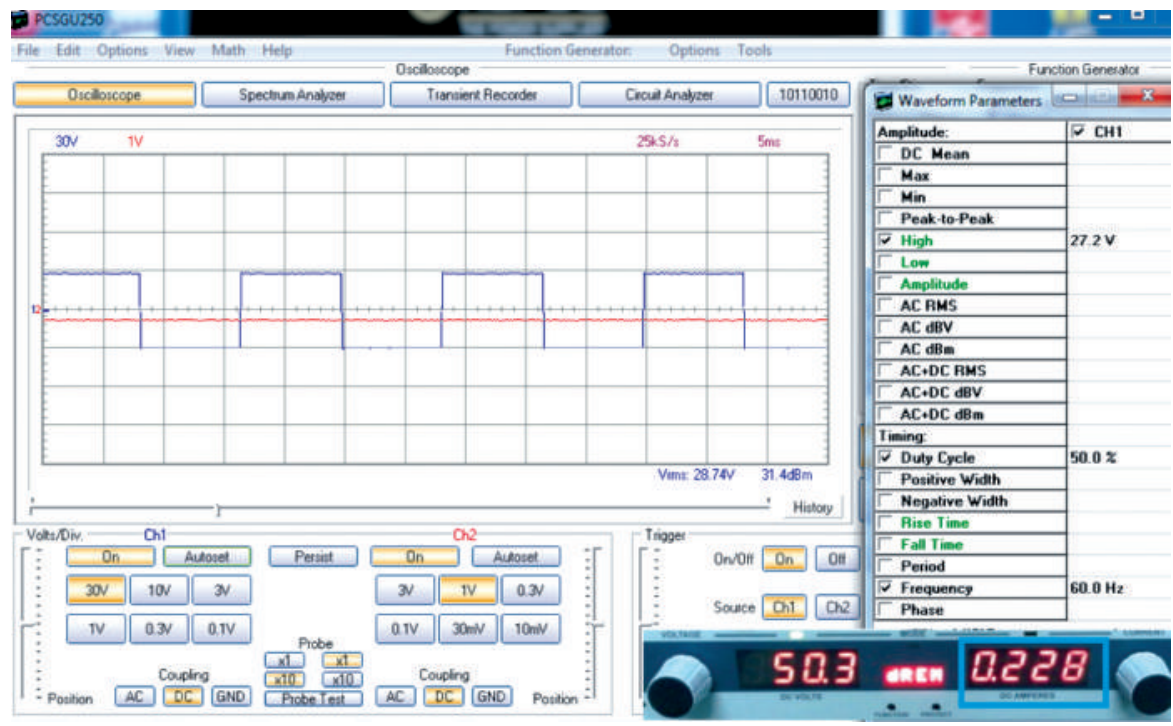

Figura 21. Variación en la carga $R=180 \Omega$.

El hecho de tener un puente $\mathrm{H}$ no significa que cada brazo conmuta completamente inverso al otro, en este caso, cada MOSFET conmuta de una manera única y esto es debido al protocolo de conmutación propuesto.

Para el diseño del convertidor fue necesario tomar en cuenta dos aspectos importantes: primero, que el convertidor es un reductor $y$, segundo, que actúa a la vez como filtro pasa bajos para eliminar la señal del control por modos deslizantes. Esta señal se encuentra en un rango de $1 \mathrm{kHz}$ a $30 \mathrm{kHz}$. El filtro pasa bajos se realiza con la finalidad de obtener únicamente la señal de $60 \mathrm{~Hz}$ requerida como parte de la aplicación.

\section{AGRADECIMIENTOS}

Los autores agradecen las facilidades prestadas en el laboratorio de Control Industrial, así como el equipamiento instrumental de la Universidad de las Fuerzas Armadas - ESPE del Ecuador que han servido de soporte para el desarrollo del presente trabajo.

\section{REFERENCIAS BIBLIOGRÁFICAS}

[1] Avilés, O., Miño, P., y Solaque L. (2002). Identificación de parámetros de sistemas dinámicos. Revista Ciencia e Ingeniería Neogranadina, 12, 41-52.

[2] Solé, D.B.(1999). Control en modo deslizante aplicado a la generación de señal en convertidores conmutados DC/ DC (Tesis doctoral inédita). Universidad Politécnica de Cataluña, Barcelona, España.

[3] Mohan, N. (2009). Electrónica de potencia: convertidores, aplicaciones y diseño. McGraw-Hill. 
[4] De la Parte, M. (2005). Control Predictivo con Modos Deslizantes (Tesis doctoral inédita). Universidad de La Rioja, Logroño, España.

[5] Colvero, A., Campos, A., Marco, A. Dalla Costa y Alonso, J.M. (2013). Interleaved Buck Converter Applied to High-Power HID Lamps Supply: Design, Modeling and Control. IEEE TRANSACTIONS ON INDUSTRY APPLICATIONS, 49(4), 18441853.

[6] Labbe, B., Allard, B. y Xuefang, L.-S. (2014). Design and Stability Analysis of a Frequency Controlled Sliding-Mode Buck Converter. Circuits and Systems I: Regular Papers, IEEE Transactions, 61(9), 2761-2770.

[7] Gautam, A.R., Mahela, O.P., Singh, S., Mahia, R.N. (2014). A double input SMV controlled DC/DC buck-boost converter for solar PV/wind energy sources. Recent Advances and Innovations in Engineering (ICRAIE). Jaipur, India: IEEE.

[8] Afsharian Zadeh, A. y Khayatian, A. (2014). Derivative-integral terminal sliding-mode control strategy for DC-DC buck converters. Power Electronics, Drive Systems and Technologies Conference (PEDSTC), 5. Teherán, Irán: IEEE.

[9] Yue, Z., Wei, Q. y Daihyun, H. (2014). A Sliding-Mode Duty-Ratio Controller for DC/DC Buck Converters With Constant Power Loads. Industry Applications, IEEE Transactions, 50(2), 1448-1458.

[10] Gamboa, J.L. (2008). Guía de diseño y análisis de convertidores conmutados de alta frecuencia (Tesis de pregrado inédita). Universidad de Costa Rica, Costa Rica.

[11] De la Fuente Méndez, G. (2009). Análisis de una fuente de poder conmutada (Tesis de pregrado inédita). Universidad Veracruzana, Veracruz, México.

[12] Battista, H.D. (2000). Control de la calidad de potencia en sistemas de conversión de energía eólica (Tesis doctoral inédita). Universidad Nacional de La Plata, La Plata, Argentina.

[13] Domingo Biel, E.F. y Olm, J.M. (2007). Robust Step-up DC/AC conversion with a Full-Bridge Non-Inverting Buck-Boost. 16th IEEE International Conference on Control Applications. 1 (pp.593-598). Singapur: IEEE.

[14] Sira-Ramírez, H. y Silva-Ortigoza, R.(2006). Control Design Techniques in Power Electronics Devices. Springer.

[15] Utking, V.I. (1977). Variable Structure Systems with Sliding Mode. IEEE Transactions on Automatic Control, 22, 212-222.

[16] Sira-Ramírez, H. (2005). Control de Sistemas no lineales. PEARSON EDUCATION, S.A.

[17] Martínez, C.A.O. (2003). Control de un conversor de potencia con modulador de ancho de pulso centrado usando modos deslizantes (Tesis de maestría inédita). Universidad Nacional de Colombia, Bogotá, Colombia. 